Frequency Swept Microwaves for Hyperfine

Leave this area blank for abstract info.

Decoupling and Time Domain Dynamic

Nuclear Polarization

Daniel E. M. Hoff, Brice J. Albert, Edward P. Saliba, Faith J. Scott, Eric J. Choi, Michael Mardini, and Alexander B. Barnes
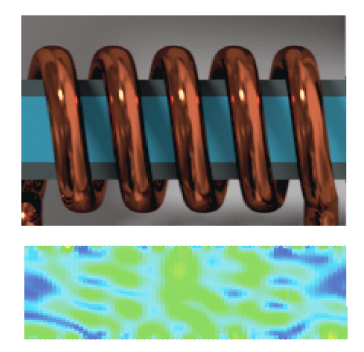

time independent microwave calculations

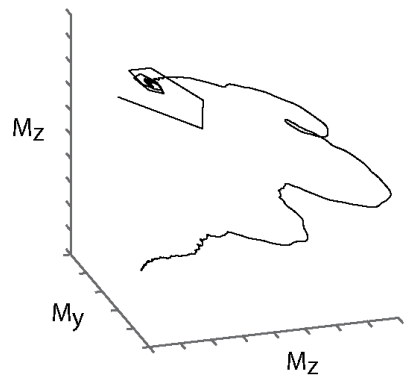

adibatic EPR inversions underMAS

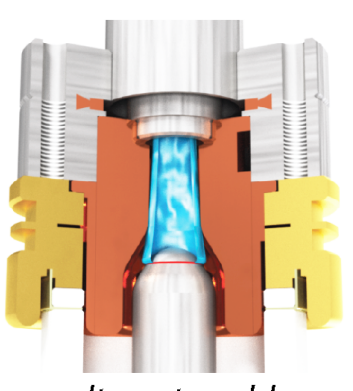

voltage tunable gyrotrons 
Solid State Nuclear Magnetic Resonance

\title{
Frequency Swept Microwaves for Hyperfine Decoupling and Time Domain Dynamic Nuclear Polarization
}

\author{
Daniel E. M. Hoff, Brice J. Albert, Edward P. Saliba, Faith J. Scott, Eric J. Choi, Michael Mardini, and \\ Alexander B. Barnes* \\ Department of Chemistry, Washington University in St. Louis, One Brookings Drive, St. Louis MO 63130, USA
}

\begin{abstract}
ARTICLE INFO
ABSTRACT

Article history:

Received

Received in revised form

Accepted

Available online

Keywords:

Dynamic Nuclear Polarization

Hyperfine Decoupling

Electron Decoupling

Pulsed DNP

Voltage Tunable Gyrotrons

Magic Angle Spinning

Frequency Modulated Cross Effect

Dielectric Heating

Adiabatic Inversion

Hyperfine decoupling and pulsed dynamic nuclear polarization (DNP) are promising techniques to improve high field DNP NMR. We explore experimental and theoretical considerations to implement them with magic angle spinning (MAS). Microwave field simulations using the high frequency structural simulator (HFSS) software suite are performed to characterize the inhomogeneous phase independent microwave field throughout a $198 \mathrm{GHz}$ MAS DNP probe. Our calculations show that a microwave power input of $17 \mathrm{~W}$ is required to generate an average EPR nutation frequency of $0.84 \mathrm{MHz}$. We also present a detailed calculation of microwave heating from the HFSS parameters and find that $7.1 \%$ of the incident microwave power contributes to dielectric sample heating. Voltage tunable gyrotron oscillators are proposed as a class of frequency agile microwave sources to generate microwave frequency sweeps required for the frequency modulated cross effect, electron spin inversions, and hyperfine decoupling. Electron spin inversions of stable organic radicals are simulated with SPINEVOLUTION using the inhomogeneous microwave fields calculated by HFSS. We calculate an electron spin inversion efficiency of $56 \%$ at a spinning frequency of $5 \mathrm{kHz}$. Finally, we demonstrate gyrotron acceleration potentials required to generate swept microwave frequency profiles for the frequency modulated cross effect and electron spin inversions.
\end{abstract}

\section{Introduction}

Dynamic nuclear polarization (DNP) increases NMR sensitivity by transferring polarization from electron to nuclear spins [1]. In a series of seminal papers published in the early 1990's, Jacob Schaefer and his colleagues applied DNP to magic angle spinning (MAS) NMR experiments of polystyrene samples doped with stable organic radicals [2-4]. In the years since Schaefer's first experiments, MAS DNP has exploded in popularity [5-10]. Gains in NMR sensitivity from MAS DNP are approaching the theoretical limit of 660 , which is determined by the gyromagnetic ratios of electron and proton spins [11-13]. Developments in high-power microwave sources and MAS DNP probe technology by Griffin and his colleagues at M.I.T. have played a critical role in the extension of DNP from field strengths of 1.4 Tesla employed in Schaefer's original MAS DNP to experiments at 18.7 Tesla [14-17]. Further advances in instrumentation are required to implement pulsed DNP and hyperfine decoupling schemes.

Pulsed techniques yield significant advantages over continuous wave irradiation to control and measure interactions in the solid state NMR Hamiltonian. As Schaefer demonstrated in his development of double resonance MAS NMR, short, powerful pulses permit the measurement of weak heteronuclear dipolar couplings (REDOR) [18], and also polarization transfer through weak dipolar couplings (TEDOR) [19]. Pulsed EPR techniques are also advantageous for DNP.

Performing high field DNP in the time domain can substantially improve electron to nuclear polarization transfer $[20,21]$. For example, modulation of the microwave frequency to increase the effective irradiation bandwidth improves DNP enhancements in cryogenic non-spinning experiments [22, 23]. Time domain DNP also yields much higher enhancements for non-cryogenic NMR. The integrated solid effect (ISE) and nuclear orientation via electron spin locking (NOVEL) are pulsed DNP experiments that yield high signal enhancements at room temperature [24-29]. However, most time domain DNP experiments have only been demonstrated at lower frequencies $(\sim 9 \mathrm{GHz})$ where intense microwave fields are readily available. Extending pulsed DNP techniques to high frequency MAS NMR requires further microwave technology development.

Another analogy between Schaefer's pioneering experiments in MAS NMR and current developments in DNP is the decoupling of spins with a larger magnetic moment after the polarization transfer period. Proton decoupling after cross polarization $(\mathrm{CP})$ is often critical to extending the relaxation time of the observed nuclei and providing high-resolution NMR spectra, as was demonstrated in Schaefer's original CPMAS experiments [30]. Paramagnetic relaxation effects (PREs) in 
MAS DNP experiments [31, 32] could be similarly attenuated with electron decoupling after the DNP period, as proposed by Corzilius et al. [33].

Electron decoupling, also known as hyperfine decoupling and dynamic decoupling, was introduced by Jeschke and Schweiger in the context of pulsed EPR [34] and has been applied extensively to lengthen EPR relaxation times in nitrogen vacancy (NV) centers for experiments aimed at quantum computation [35, 36]. In addition to improving spectral resolution and extending nuclear relaxation times, electron decoupling in MAS DNP could also improve the polarization enhancement of bulk spins by yielding control of the spin diffusion barrier. The spin diffusion barrier impedes the spread of enhanced proton polarization around the DNP polarizing agent $[37,38]$. Protons with strong hyperfine couplings could first be polarized through DNP, followed by an electron decoupling period during which the hyperfine couplings are attenuated and the polarized protons are brought into spin diffusion contact with bulk protons.

We discuss in the following sections how voltage tunable gyrotron oscillators could generate the microwave frequency profiles required for the inversion of electron spins and thus decoupling of hyperfine interactions. In particular, we show that EPR inversions are theoretically possible even with the inhomogeneous microwave field present within MAS rotors, and perform detailed electromagnetic simulations to characterize the microwave field intensity in a MAS DNP probe operating at 198 $\mathrm{GHz}$. We also demonstrate modulations of the gyrotron acceleration potential suitable for the frequency modulated cross effect and electron spin inversions.

\section{Microwave Field Calculations}

We used Ansys HFSS (high frequency structural simulator) to calculate the $198 \mathrm{GHz}$ electromagnetic field structure within our custom MAS DNP probe [39]. By solving for the phase independent field intensities, we are able to determine the dielectric sample heating and inhomogeneous $\gamma_{S} B_{1 S}$ frequencies, where $B_{1 S}$ represents the microwave field orthogonal to the main magnetic field.

\subsection{Microwave simulation geometry and parameters}

We calculated the electromagnetic field structure for the sample geometry shown in Fig. 1a. Inside the stator is a 5-turn RF coil with a wire diameter of $0.686 \mathrm{~mm}$. The rotor sheath is a sapphire cylinder with an outside diameter of $3.175 \mathrm{~mm}$ and has a wall thickness of $0.55 \mathrm{~mm}$. Further details of the model geometry are given in the supplementary information. We assumed that all microwave power leaving the volume shown in Fig. 1a was lost to its surroundings. Our simulation models the sample as a frozen glycerol and water mixture. We simulate the effect of the sample on the $198 \mathrm{GHz}$ field structure by setting the real part of the dielectric constant $\left(\varepsilon_{r}^{\prime}\right)$ to 3.5 and the loss tangent to 0.007 , as previously determined by Nanni et al.[39]. The relative dielectric constant is defined as:

$$
\varepsilon_{r}=\frac{\varepsilon^{\prime}+i \varepsilon^{\prime \prime}}{\varepsilon_{0}}
$$

Our simulations are normalized to microwave power levels we expect to achieve with our custom $198 \mathrm{GHz}$ voltage tunable gyrotron, designed in collaboration with Bridge 12 Technologies (Waltham, MA). We modeled the microwave input into the stator as a pure Gaussian beam with a waist of $3.175 \mathrm{~mm}$ and power of $5.0 \mathrm{~W}$. 


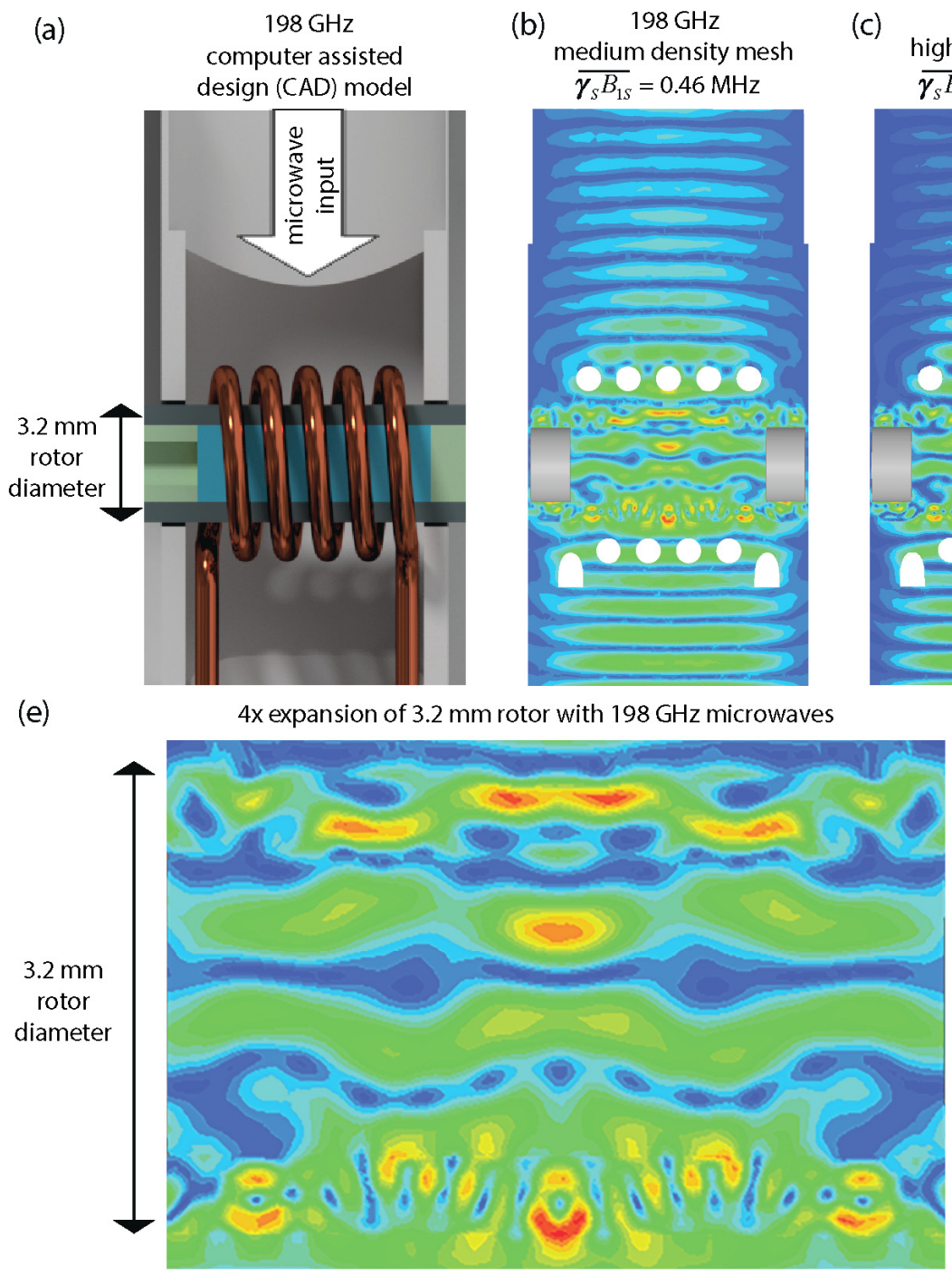

$198 \mathrm{GHz}$ high density mesh $\overline{\gamma_{S} B_{1 S}}=0.46 \mathrm{MHz}$

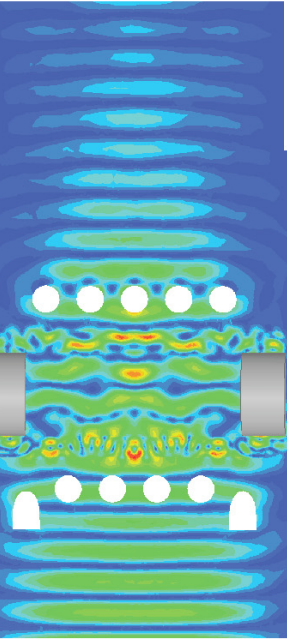

(d) legend: instantaneous magnetic field intensity $5.0 \mathrm{~W}$ of input power and coordinate axis system from HFSS

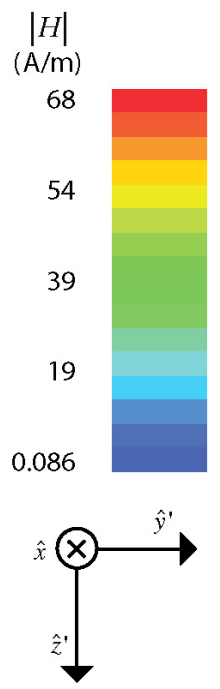

\section{(f) $4 \mathrm{~mm}$ rotor with $250 \mathrm{GHz}$ microwaves $\overline{\gamma_{S} B_{1 S}}=0.37 \mathrm{MHz}$}

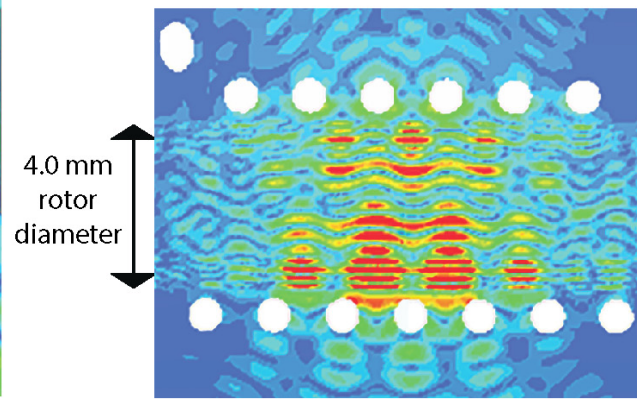

Fig. 1. Simulations of the instantaneous magnetic field magnitude in the y'-z' plane. These intensities are not representative of the $\gamma_{S} B_{1 S}$ inhomogeneity as they are dependent on time. (a) A computer assisted design (CAD) representation of our MAS DNP probe. (b) A simulation with a Gaussian beam incident on the sample. The mesh in this simulation has 15,000 tetrahedra within the sample volume. (c) The same simulation as (b) but with a higher mesh of 48,000 tetrahedra in the sample. The microwave distribution is almost identical, suggesting the calculation has converged with respect to tetrahedral density within the mesh. (d) A scale with the magnetic field magnitude normalized to $5 \mathrm{~W}$ of input power. (e) An expansion of the simulation of (c) shows considerable fine structure within the sapphire. (f) Simulation of a $250 \mathrm{GHz}$ microwave field structure on the model geometry given in Nanni et al. [39]. The qualitative structures are very similar to Nanni et al. The mesh in the simulation shown above has 94,000 tetrahedra in the sample volume.

\subsection{Calculation of $\gamma_{S} B_{1 S}$}

HFSS solves the electromagnetic field structure with an initial excitation amplitude of $\left|E_{0}\right|$. Only the initial electric field magnitude, $\left|E_{0}\right|$, needs to be given because the magnetic component, $\left|H_{0}\right|$, is proportional and orthogonal to $\left|E_{0}\right|$ for an electromagnetic wave traveling in free space. We calculated the initial electric field magnitude for our Gaussian beam propagating in free space and carrying $5 \mathrm{~W}$ of power (see supplementary information):

$$
\left|E_{0}\right|=\sqrt{\frac{4 P_{0}}{\pi \varepsilon_{0} c w_{0}^{2}}}=15,425 \mathrm{~V} / \mathrm{m}
$$

where $\varepsilon_{0}$ is the permittivity of free space, $c$ is the speed of light, and $w_{0}=3.175 \mathrm{~mm}$ is the beam waist of the Gaussian beam entering the stator.

To determine the $\gamma_{S} B_{1 S}$ within a specified volume, we first calculate the magnitude of the transverse components of the electromagnetic field. However, the HFSS calculation is not performed in the laboratory frame. We must account for this rotation of the HFSS coordinate axis from the laboratory frame to determine the magnitude of the magnetic field components orthogonal to the static field $B_{0}$ that contribute to the $\gamma_{S} B_{1 S}$. Referring to Fig. 2a we see that in the primed coordinate frame (HFSS simulation axis):

$$
\hat{y}=\left(\begin{array}{c}
0 \\
\sin \left(\theta_{m}\right) \\
\cos \left(\theta_{m}\right)
\end{array}\right)
$$


$\hat{x}^{\prime}=-\hat{x}$, and $\theta_{m}$ is the magic angle. Note that $\hat{z}^{\prime}$ is the incident

axis of the Gaussian beam and $\hat{y}^{\prime}$ is the long rotor axis of the sample (see Fig. 2a).

We define the physically significant quantity for EPR and DNP to be the volume average of the peak transverse magnetic field similar to the treatment in Macor et al. [40]:

$$
\left\langle B_{\perp}\right\rangle=\frac{1}{V} \int_{V}\left|\mathbf{B}_{\perp}\right| d V=\frac{\mu}{V} \int_{V} \sqrt{|\mathbf{H} \cdot \hat{x}|^{2}+|\mathbf{H} \cdot \hat{y}|^{2}} d V
$$

Note, this differs slightly from the procedure outlined in Nanni et al. in which the volume root mean square (RMS) is computed [39].

We also assume $\mu$ is constant throughout the entire sample and is approximated by the permeability of free space, $\mu_{0}=1.257 \times 10^{-6} \mathrm{H} / \mathrm{m}$. The permeability, $\mu$, is a measure of the response of the material to an applied magnetic field in the similar way that the dielectric constant, $\varepsilon$, is the response of a material to an applied electric field. The detailed calculations including all numerical values and screen-shots from HFSS are given in the supplementary information.

When we integrate over the entire sample volume of 18.6 $\mu \mathrm{L}$ we find that $5.0 \mathrm{~W}$ of power gives an average $\gamma_{S} B_{1 S}$ of 0.46 MHz. $17 \mathrm{~W}$ of input power into the stator is required to generate the average $\gamma_{S} B_{1 S}$ of $0.84 \mathrm{MHz}$ which is now commonly used in theoretical treatments of MAS DNP. [41, 42]

\subsection{Consideration of linear vs. circularly polarized light}

The microwave beam emitted from gyrotrons is linearly polarized, however electron spins only strongly interact with circularly polarized light. We can decompose the linearly oscillating field into two circularly polarized components that each have half the amplitude of the original beam as shown in Fig. 2b. The component rotating with the spins contributes to the nutation; the other can be neglected. This means that the average $B_{1 S}$ over the sample given is $B_{\perp} / 2$. It should be noted that if $B_{\perp}$ is given as the RMS, $B_{\perp, R M S}$ of a linearly oscillating field then, $B_{1 S}=B_{\perp, R M S} / \sqrt{2}$. This is the treatment given in Nanni et al.[39] and Macor et al.[40].

\subsection{Calculating dielectric sample heating}

A concern often voiced about MAS DNP using high power microwaves is heating of the sample. This has already been calculated from similar electromagnetic field simulations [39, 40]. For dielectric heating the generated power density per volume is:

$$
W=\frac{1}{2} \omega \varepsilon_{r}^{\prime \prime} \varepsilon_{0}|\mathbf{E}|^{2}
$$

where $\omega$ is the angular frequency of the incoming linearly polarized light. The power transferred to heat for a given volume of dielectric material is the integral:

$$
\int_{V} W d V=\frac{1}{2} \omega \varepsilon_{r} \varepsilon_{0} \varepsilon_{V}|\mathbf{E}|^{2} d V
$$

Using HFSS we solved this integral and found that $7.1 \%$ of the power goes into heating the sample for our $198 \mathrm{GHz}$ system. As previously discussed, the $\sim 1.0 \mathrm{~W}$ of power that contributes to dielectric heating is effectively dissipated in MAS DNP

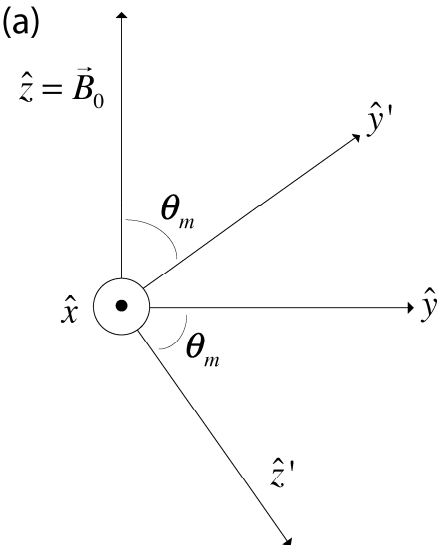

(b)

Fig. 2. (a) Coordinate axis of simulation relative to the main magnetic field. $\hat{z}^{\prime}$ is the incident axis of the Gaussian beam in the simulation, $\hat{y}^{\prime}$ is the rotor axis and $\theta_{m}=54.74^{\circ}$. (b) decomposition of a linearly polarized magnetic vector into two oppositely rotating vectors with $1 / 2$ the magnitude.

experiments by the sapphire rotor which is cooled by a high flow of cryogenic gas $[13,39,43]$.

\subsection{Comparison to microwave fields at $250 \mathrm{GHz}$}

To determine the effect of geometry and frequency on the microwave field structure we applied our same analysis to the 4.0 mm DNP NMR probe modeled by Nanni et al. at $250 \mathrm{GHz}$ as shown in Fig. 1f. The field structures are considerably different with the $3.2 \mathrm{~mm}$ rotor at $198 \mathrm{GHz}$, and the $4 \mathrm{~mm}$ rotor at 250 GHz. Calculating the microwave structure at the appropriate geometry and frequency are important aspects to understanding and optimizing MAS DNP performance.

The microwave intensities in the $4 \mathrm{~mm}$ rotor at $250 \mathrm{GHz}$ are qualitatively similar to the structures seen previously, although we cannot directly compare the values because the stator geometries are not identical. The values in Nanni et al. include a silver coating applied to the inside of the stator that we have not modeled. The analysis given by Nanni et al. reports $\gamma_{S} B_{1 S}$ values of $0.84 \mathrm{MHz}$ for a silver coated stator, and $0.70 \mathrm{MHz}$ without the silver coating.

With $5 \mathrm{~W}$ of input power, we found an average value $\gamma_{S} B_{1 S}$ of $0.37 \mathrm{MHz}$ within the sample at $250 \mathrm{GHz}$. However, if we take a volume RMS, as outlined by Nanni et al., we determine a RMS value of $0.46 \mathrm{MHz}$ within the sample volume. This agrees well with the value of $0.49=0.70 / \sqrt{2} \mathrm{MHz}$, which accounts for the circularly polarized component at $250 \mathrm{GHz}$.

We calculated the standard deviation of the peak transverse magnetic field normalized to the mean transverse magnetic field within the sample as:

$$
\hat{\sigma}=\frac{\sqrt{\left\langle B_{\perp}{ }^{2}\right\rangle-\left\langle B_{\perp}\right\rangle^{2}}}{\left\langle B_{\perp}\right\rangle}
$$

The more homogenous $\gamma_{S} B_{1 S}$ distribution will have a standard deviation closer to 0 . For our $198 \mathrm{GHz}$ system we found $\hat{\sigma}=0.28$. For the $250 \mathrm{GHz}$ system outlined we found $\hat{\sigma}=$ 0.75 . This suggests that the transverse magnetic field is more homogenous within the sample for our $198 \mathrm{GHz}$ system than the $250 \mathrm{GHz} / 4 \mathrm{~mm}$ probe absent of a silver coating applied to the inside of the stator. 
2.6 Instantaneous microwave field compared to $\gamma_{S} B_{1 S}$

The instantaneous microwave intensities shown in Fig. 1 do not accurately represent the $\gamma_{S} B_{1 S}$ distribution. This is because the microwave field intensity is time dependent and follows the 5 picosecond oscillation period of the $198 \mathrm{GHz}$ microwaves. Fig. 3a clearly shows the microwave field intensity within the sample is strongly dependent on the phase of the microwaves as they propagate. We show a time dependent microwave field structure and the time independent $\gamma_{S} B_{1 S}$ distribution in Fig. 3b. The time (and phase) independent field structure is similar to the field structures shown in Nanni et al. [39]. Clearly the $\gamma_{S} B_{1 S}$ distribution is much more homogeneous than the instantaneous intensity of the microwaves. However, we note there is still a considerable inhomogeneity in the $\gamma_{S} B_{1 S}$ frequencies, which we account for in our simulations of EPR inversions in section 4 .

\section{Experimental Considerations for MAS Pulsed DNP}

To give insight into the experimental requirements for performing MAS pulsed DNP, we calculated the amount of microwave power necessary to generate a $\pi / 2$ pulse on the electron spin manifold. At 7 Tesla, the inhomogeneously broadened nitroxide lineshape simulated with EasySpin [44] is approximately $750 \mathrm{MHz}$ broad [45]. The $\gamma_{S} B_{1 S}$ frequency required to control this lineshape with a short $(\sim 1 \mathrm{~ns})$ hard pulse is thus $\sim 750 \mathrm{MHz}$ and would require an $\left|E_{0}\right|$ value of $4.5 \times 10^{7}$

$\mathrm{V} / \mathrm{m}$, which corresponds to a microwave input power of 13 megawatts. Impressively, gyrotrons built for fusion research approach such power levels but do not generate more than 1.3 megawatts [46, 47]. Using equation (6), we calculated the amount of dielectric heating in the sample, if it were possible to irradiate with 13 megawatts, to be $940 \mathrm{~kW}$. Thus, increasing the microwave power to generate short hard pulses to excite the entire nitroxide lineshape at magnetic fields above 7 Tesla is not experimentally feasible with current MAS DNP microwave coupling strategies.

One strategy for MAS pulsed DNP at high magnetic fields is to implement a microwave resonant structure with a high quality factor similar to high field non-spinning DNP probes [48, 49]. For instance, an EPR cavity with a quality factor of 200 would still provide the frequency bandwidth sufficient to cover the entire nitroxide lineshape, but reduce the input power requirement from 13 megawatts to $67 \mathrm{~kW}$. An additional advantage to such microwave resonance structures is the ability to separate the magnetic field component of the microwave field required to manipulate the electron spins from the electric component responsible for dielectric heating, as shown in equations (4) and (6). However, a microwave resonator with a quality factor of 200 compared to the current quality factors in MAS DNP probes which are near unity, would be extremely hard to implement given the experimental requirements of MAS. For instance, the relatively large sample size, the RF coil, and the rotor lead to much lower microwave quality factors and worse coupling of the electromagnetic power into the sample than encountered in typical NMR and EPR experiments.

Stable organic radicals with more symmetric g-tensors, such as 1,3-bis(diphenylene)-2-phenylallyl (BDPA) and trityl [50], have major advantages as polarizing agents for pulsed DNP over nitroxides, including less inhomogeneous broadening of the EPR lineshapes and much longer electron spin relaxation times [43]. The long longitudinal relaxation $\left(T_{1 S}\right)$ [51] and narrow linewidth $(\sim 30 \mathrm{MHz}$ at $140 \mathrm{GHz}$ [52]) of BDPA made it an excellent DNP polarizing agent used by Schaefer, Griffin, and their colleagues to yield significant signal enhancements at room temperature in some of the first MAS DNP experiments $[2,14]$. More recently trityl and a sulfonated water-soluble analog of BDPA (SA-BDPA) have been well characterized as excellent DNP polarizing agents for the solid effect and Overhauser effect [52-56].

Again, we apply the results from our HFSS microwave field calculations and determine that $21 \mathrm{~kW}$ of microwave power are required to generate a $30 \mathrm{MHz} \gamma_{S} B_{1 S}$ field to effectively excite the BDPA lineshape with a hard microwave pulse of $\sim 33$ nanoseconds. Similar to the previous discussion, $110 \mathrm{~W}$ of power would be required if a microwave cavity with a quality factor of 200 was implemented. Our laboratory is engaged in designing such MAS microwave resonators. In the following sections, we propose strategies that can be implemented with the inhomogeneous $\gamma_{S} B_{1 S}$ frequencies currently available to perform time domain DNP and hyperfine decoupling with MAS.

\section{Simulations of EPR Inversions}

The adiabatic full passage, or adiabatic inversion is a technique in which the magnetization of the sample is slowly swept 180 degrees from $+S_{z}$ to $-S_{z}[57,58]$. Adiabatic pulses can yield broadband decoupling [59-62], even when the $\gamma B_{1}$ (a)

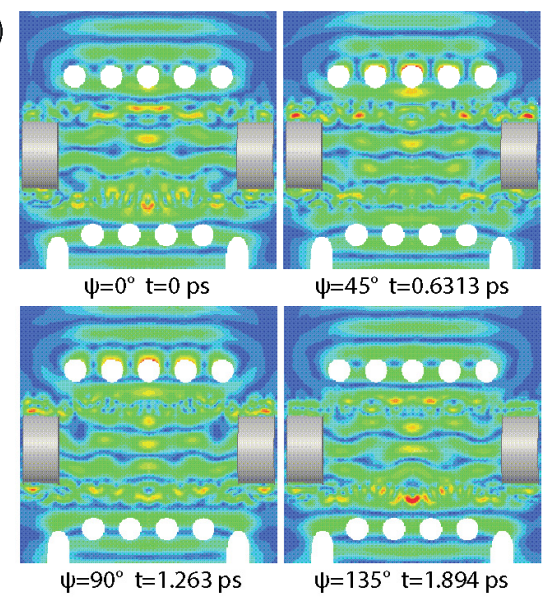

(b)

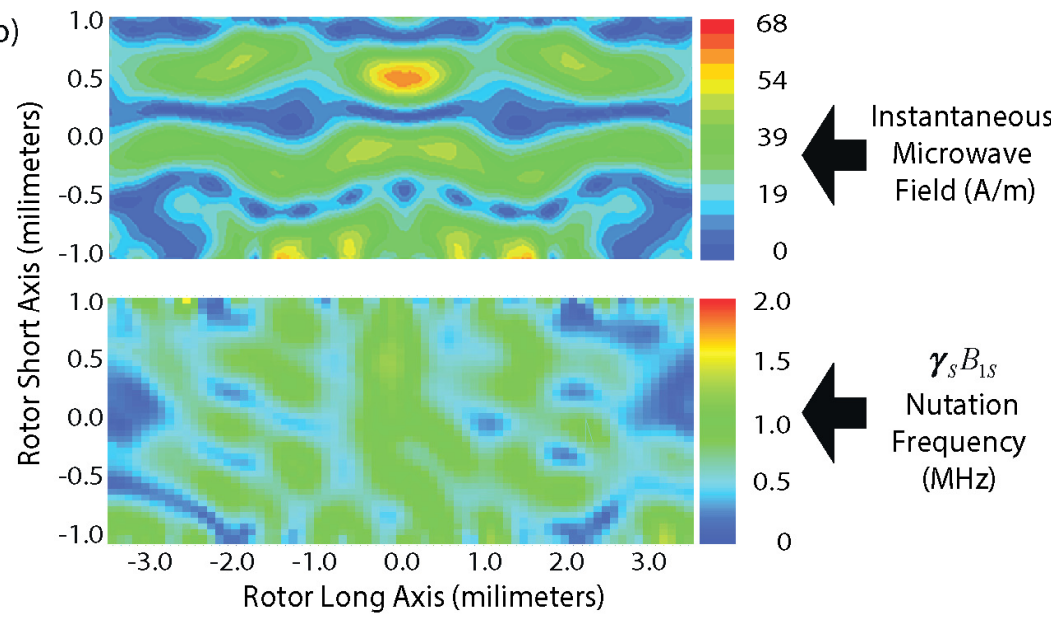

Fig. 3. (a) Phase dependence of the microwave fields within the sample (b) comparison of a time dependent microwave field structure (top) and time independent $\gamma_{S} B_{1 S}$ distribution (bottom). 
frequency is much less than the spin resonance linewidth, as is currently the case for EPR in MAS DNP experiments. Adiabatic spin inversions can be accomplished by modulating the frequency of the RF (or microwave) field according to linear, tangential, and hyperbolic secant functions, among others. During the frequency sweep, the magnetization is largely locked along the direction of the effective field, provided the sweep rate is optimized, the $\gamma_{S} B_{1 S}$ is the appropriate frequency, and the relaxation times are long enough. One major advantage of adiabatic sweeps over pulsed magnetic resonance is that given a sufficiently strong microwave field, the inversion efficiency is essentially independent of $B_{1}$ inhomogeneities $[57,58]$.

\subsection{Conditions for an adiabatic inversion}

When performing an adiabatic inversion, one comes up against two competing phenomena as described by Kupce and Freeman [57]. The effective field must be swept slowly enough that the magnetization of the sample is locked along it. However, if it is varied too slowly the extent of relaxation, particularly via spin-spin relaxation, becomes prohibitively large. The first condition is commonly described by a parameter known as the "adiabaticity factor" and is defined according to the following equation [57]:

$$
Q(t)=\left|\frac{\omega_{e f f}(t)}{\frac{d \theta(t)}{d t}}\right|
$$

Here $\omega_{\text {eff }}(t)$ is the effective field in units of angular frequency, and $d \theta(t) / d t$ is the rate at which the angle of the effective field with the $\mathrm{x}-\mathrm{y}$ plane is changing with time. A useful form of this equation can be realized by recognizing that $\omega_{\text {eff }}(t)$, the frequency offset, $\Delta \omega(t)$, and the strength of the microwave field, $\omega_{1}(t)$, are related in the following manner:

$$
\omega_{\text {eff }}(t)=\left[\Delta \omega(t)^{2}+\omega_{1}(t)^{2}\right]^{1 / 2}
$$

$\theta(t)$ can also be written in terms of the frequency offset and microwave strength:

$$
\theta(t)=\tan ^{-1}\left(\frac{\Delta \omega(t)}{\omega_{1}(t)}\right)
$$

Differentiating with respect to time yields:

$$
\frac{d \theta(t)}{d t}=\frac{\omega_{1}(t) \frac{d \Delta \omega(t)}{d t}-\Delta \omega(t) \frac{d \omega_{1}(t)}{d t}}{\omega_{1}(t)^{2}+\Delta \omega(t)^{2}}
$$

If we now rewrite $Q(t)$ in terms of $\Delta \omega(t)$ and $\omega_{1}(t)$, we obtain a general formula for the adiabaticity factor in terms of experimental variables:

$$
Q(t)=\left|\frac{\left[\Delta \omega(t)^{2}+\omega_{1}(t)^{2}\right]^{\frac{3}{2}}}{\omega_{1}(t) \frac{d \Delta \omega(t)}{d t}-\Delta \omega(t) \frac{d \omega_{1}(t)}{d t}}\right|
$$

In the special case of a linear sweep, which is the sweep function simulated in section 4.3, the frequency offset is modulated as a function of time according to the following equation:

$$
\Delta \omega(t)= \pm k\left(t-\frac{1}{2} \tau\right)
$$

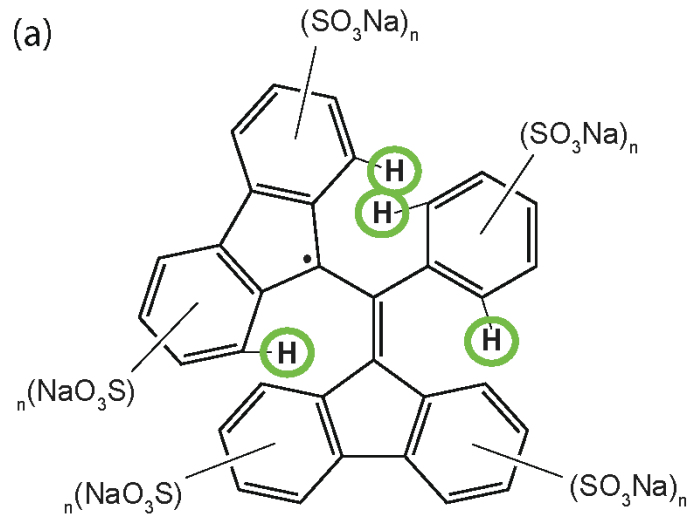

(b)

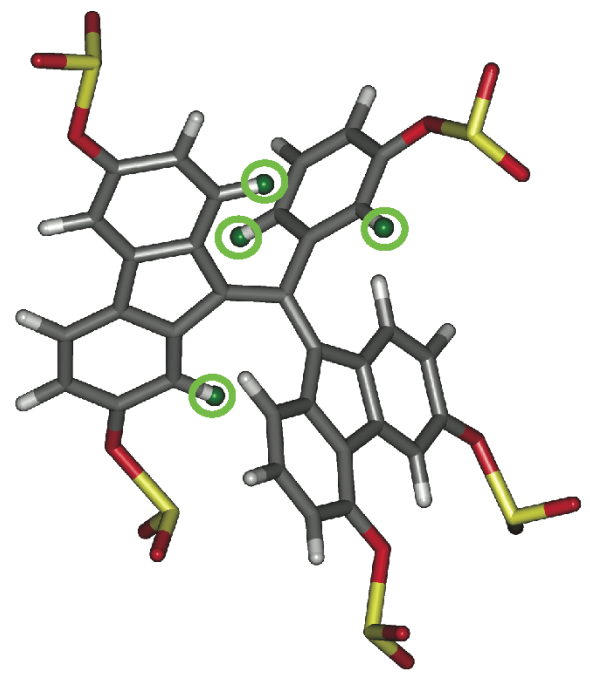

(c)
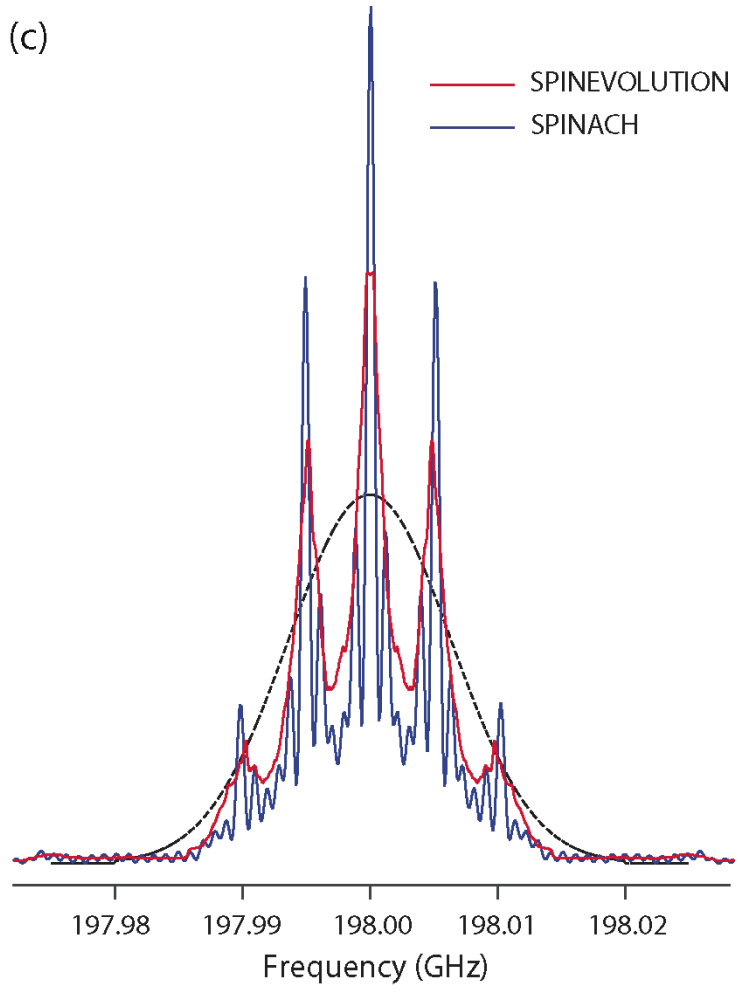

Fig. 4. Sulfonated BDPA (a) Chemical structure and (b) Energy minimized structure from GAMESS[63], indicating the 4 protons with strong hyperfine interactions included in the spin simulations (c) Simulated EPR spectrum of sulfonated-BDPA from SPINEVOLUTION in red, SPINACH in blue, and a Lorentzian profile in dotted black that matches closely to experiment [56]. 
(a)

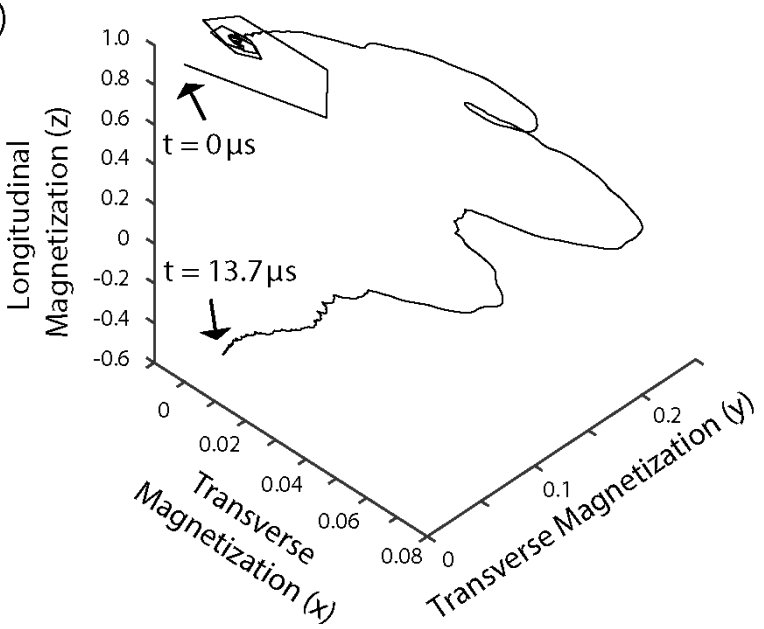

(c)

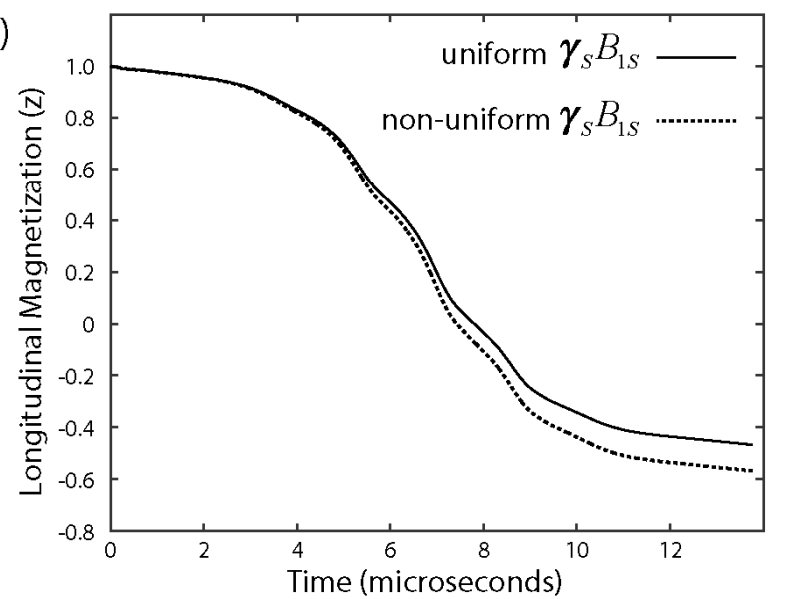

(b)

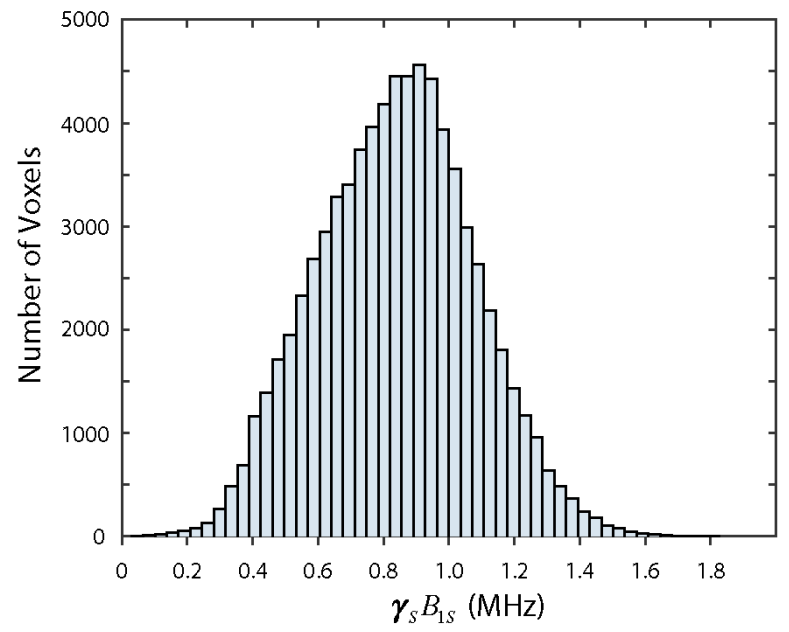

(d)

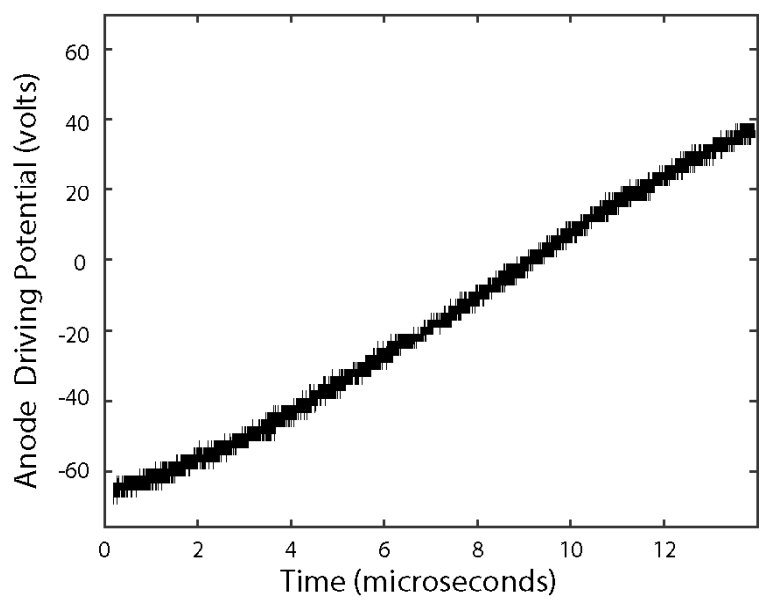

Fig. 5. Adiabatic EPR inversions for MAS DNP. (a) Simulation of the EPR magnetization during an adiabatic inversion with a homogeneous $\gamma_{S} B_{1 S}=0.84 \mathrm{MHz}$. (b) Histogram indicating the distribution of $\gamma_{S} B_{1 S}$ frequencies in the $3.2 \mathrm{~mm}$ rotor at $200 \mathrm{GHz}$ as determined by the HFSS model. (c) The longitudinal magnetization during adiabatic inversions with a homogeneous $\gamma_{S} B_{1 S}$ (black line) and the inhomogeneous $\gamma_{S} B_{1 S}$ calculated from the HFSS model (dotted line). (d) Voltage trace from an oscilloscope showing the estimated gyrotron potential required for the electron spin inversions.

Here $k$ is the sweep rate, $\tau$ is the total time of the sweep and $t$ runs from 0 to $\tau$. Additionally, the strength of the microwave field is held constant, so $d \omega_{1} / d t=0$ and the time dependence of $\omega_{1}(t)$ can be dropped. Although there could be a substantial voltage and frequency dependence on the microwave power over a sweep range of $750 \mathrm{MHz}$ that would be required to invert a nitroxide lineshape, the microwave power over a $40 \mathrm{MHz}$ band, which is all that needed to invert a SA-BDPA resonance, is nearly constant during the frequency sweep. So for the inversion of SA-BDPA, the strength of the microwave field is assumed to be held constant. Putting these values into equation (12) renders the following for a linear sweep of the frequency offset:

$$
Q(t)=\left|\frac{\left[k^{2}(t-\tau / 2)^{2}+\omega_{1}^{2}\right]^{\frac{3}{2}}}{ \pm \omega_{1} k}\right|
$$

The larger the adiabaticity factor, the better the inversion, so we need only concern ourselves with the minimum value of this function, which occurs when $t=\frac{1}{2} \tau$. Here the adiabaticity factor is equal to the expression given by Kupce and Freeman [57]:

$$
Q_{\text {minimum }}=\frac{\omega_{1}^{2}}{k}
$$

They suggest keeping the adiabaticity factor at a value of at least 1 for NMR. This is difficult in EPR, however, because the relaxation times for an electron are substantially shorter than for a nucleus, which we take into account in our simulations.

\subsection{EPR simulations of $S A-B D P A$}

The inversion of SA-BDPA EPR spectrum was calculated using the SPINEVOLUTION simulation package [64]. We used EPR parameters previously determined at $140 \mathrm{GHz}$ of $40 \mathrm{mM}$ SA-BDPA frozen in a $\mathrm{d}_{8}$-glycerol/ $\mathrm{H}_{2} \mathrm{O} / \mathrm{D}_{2} \mathrm{O}(60 / 30 / 10) \%$ glassy matrix at 80 Kelvin determined by Smith and his colleagues [48, $56,65]$. The inhomogeneous linewidth was $30 \mathrm{MHz}$, the spin lattice $T_{1 S}$ was $56 \mathrm{~ms}$, and the phase memory time (homogeneous transverse relaxation) was $3.3 \mu \mathrm{s}$. The majority of the SA-BDPA linewidth arises from intramolecular hyperfine couplings.

Fig. 4a shows the 4 protons that were included in the simulations. There are 8 strongly coupled protons, each with a isotropic hyperfine interaction of $\sim 5.1 \mathrm{MHz}$. We included the 4 protons shown in Fig. $4 \mathrm{a}$ in the spin simulations as a representative sample due to the computational demand of including more spins. An isotropic hyperfine interaction of 5.1 
(a)

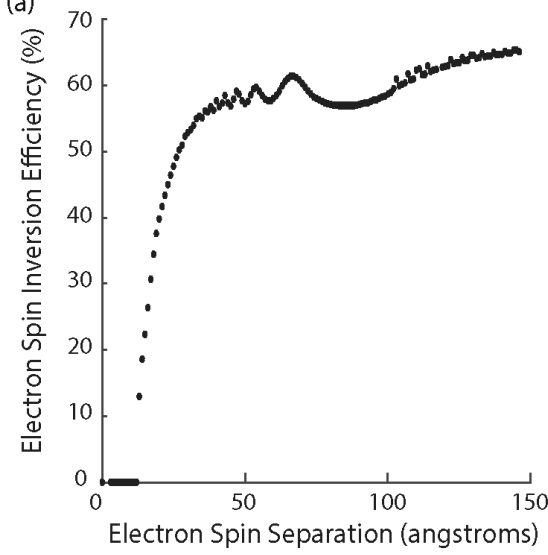

(b)

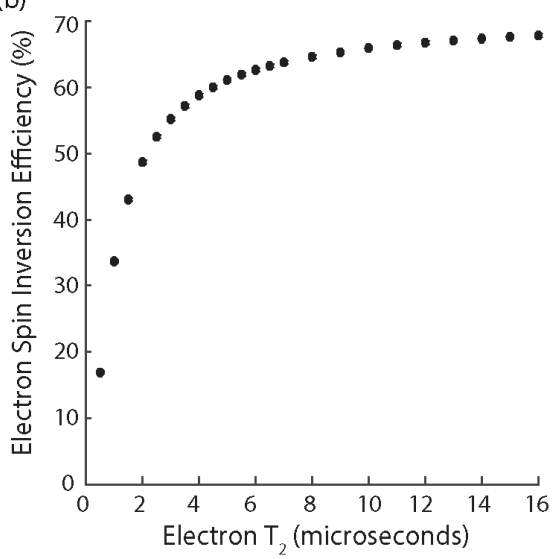

(c)

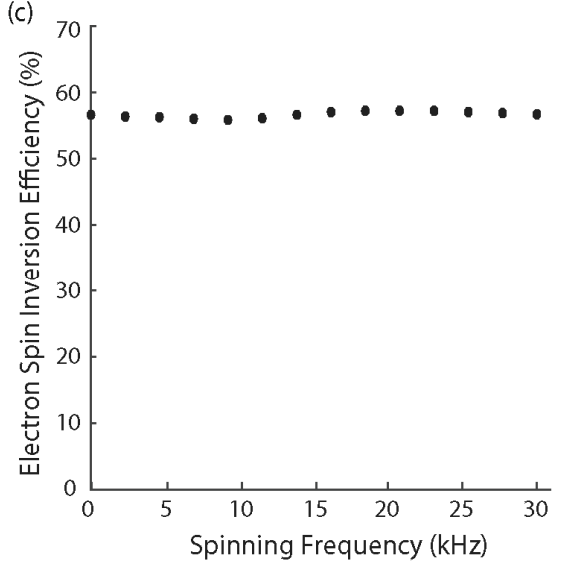

Fig. 6. The effect of various parameters on electron spin inversion efficiencies. (a) Inter-electron spin separation (b) Transverse homogenous electron relaxation time (c) Spinning frequency.

$\mathrm{MHz}$ was used in the simulations according to Haze et al.[56], and the dipolar hyperfine interaction was calculated explicitly.

In order to calculate the hyperfine dipolar interactions, the computational package GAMESS was used to obtain the relative unpaired spin densities and the atomic coordinates for each of the spins[63]. The geometry of the energy minimized SA-BDPA structure is shown in Fig. 4b. Dipolar coupling between the hydrogen spins were neglected to shorten the calculation time.

The unpaired electron of SA-BDPA is highly delocalized over a large pi network. This delocalization was approximated by repeating the simulation for 33 different electron positions. Specifically, the atomic coordinates and relative unpaired spin densities at the nuclei of each of the 33 carbons were obtained from a density functional theory calculation of SA-BDPA. The calculation was performed using the GAMESS program, with a B3LYP functional, and a $6-31 \mathrm{G}^{* *}$ basis set [63]. Spin densities were obtained via Lowdin population analysis. Simulations were carried out for each electron position, weighted by the normalized spin density, and then summed.

Fig. 4c shows results from SPINEVOLUTION compared to those from SPINACH[66]. Both results approximate the Lorentzian profile of experimental results close enough for our theoretical treatment considering our goal is to demonstrate that broadband electron spin inversions at $198 \mathrm{GHz}$ are possible with currently available microwave sources. Including more protons on the SA-BDPA molecule, along with those of the ice surrounding the molecule, would improve this agreement. Ultimately, the choice of simulation package comes to one of computation time. A five-spin system in SPINEVOLUTION is much faster with reasonable accuracy and thus allows for the exploration of more inversion parameters.

\subsection{Simulations of electron spin inversions}

To invert the electron spins of SA-BDPA, we simulated a linear frequency sweep from $20.5 \mathrm{MHz}$ below the center of the EPR resonance to $20.5 \mathrm{MHz}$ above the resonance, over a period of $13.75 \mu \mathrm{s}$. With a $\gamma_{1} B_{1 S}$ of $0.84 \mathrm{MHz}$, this yields an adiabaticity factor of 0.24 (from equation (15)). This adiabaticity factor can be improved with higher $\gamma_{1} B_{1 S}$ values. Also, more efficient sweep schemes exist than the linear sweep which yield better adiabaticity factors. These could also be implemented with frequency agile gyrotrons.

Using HFSS we computed the $\gamma_{S} B_{1 S}$ in each of 75,295 voxels in the sample. The average $\gamma_{S} B_{1 S}$ value over the entire sample was determined by averaging the values of all the voxels. This came out to a value of $0.84 \mathrm{MHz}$ with $17 \mathrm{~W}$ of power input into the MAS stator and was used to optimize the sweep parameters.

The tip of the magnetization vector's trajectory is shown in Fig. 5a. The inversion efficiency achieved with an entirely homogenous field of $0.84 \mathrm{MHz}$ was $57 \%$. In order to simulate the inhomogeneity of the microwave field, the microwave strengths generated in HFSS with an average $\gamma_{1} B_{1 S}$ of $0.84 \mathrm{MHz}$ were binned into groups. Electron spin inversions were calculated on each bin, and the results were weighted according to the population distribution shown in Fig. 5b. This process was done using both 50 and 100 bins, both of which resulted in $46 \%$ spin inversion, indicating the binning of the microwave field inhomogeneity converges at less than 50 bins.

Fig. 5c shows the time dependencies of the $z$ components of the SA-BDPA magnetization under an adiabatic inversion in both the homogeneous, average field, and the real, inhomogeneous field. The similar magnetization profiles demonstrate that frequency swept microwave pulses are a robust strategy to invert electron spins in MAS experiments. To connect our theoretical analysis to experiments, we demonstrate in Fig. $5 \mathrm{~d}$ the gyrotron acceleration profile required to produce the frequency sweep and electron spin inversion. All subsequent simulations discussed from here employ a homogeneous, average field of $\gamma_{1} B_{1 S}=0.84 \mathrm{MHz}$.

There are some factors that will attenuate the inversion percentages reported here. We first consider the dipolar interaction between electrons on nearby SA-BDPA molecules. In order to investigate this effect, inversion simulations on two SABDPA molecules as a function of inter-electron distance were performed. In the simulations, we also included the isotropic and dipolar hyperfine interactions of each electron with two protons within its own molecule. All proton-proton dipolar couplings were neglected. The effect of the inter-electron distance up to $150 \AA$ on the electron spin inversion efficiency is shown in Fig. 6a. It can be seen that there is no inversion up to about $12 \AA$ of separation, at which point the inversion efficiency achieves its maximum value rather quickly. Using a simple cubic lattice of electrons as a model, the average distance between an electron and its nearest neighbor at $40 \mathrm{mM}$ is about 35 angstroms. As can be seen from the simulation, at this distance the inversion is essentially the same as the isolated case.

Another parameter that can affect the inversion efficiency is the phase memory relaxation time. Fig. $6 \mathrm{~b}$ shows the dependence of the inversion efficiency on $T_{2}$. A substantial amount of inversion is achieved at 3.3 microseconds.

Magic angle spinning can also affect the efficiency of the inversion. Simulations were conducted between $5 \mathrm{kHz}$ and 30 $\mathrm{kHz}$ in steps of $5 \mathrm{kHz}$ and are shown in Fig. 6c. We calculated an 
(a)

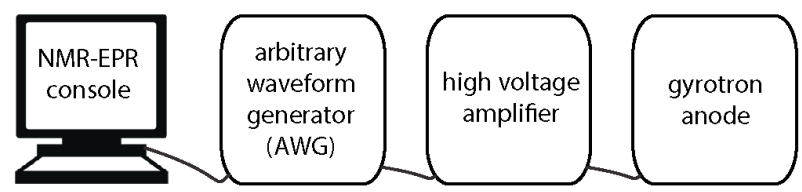

(b)

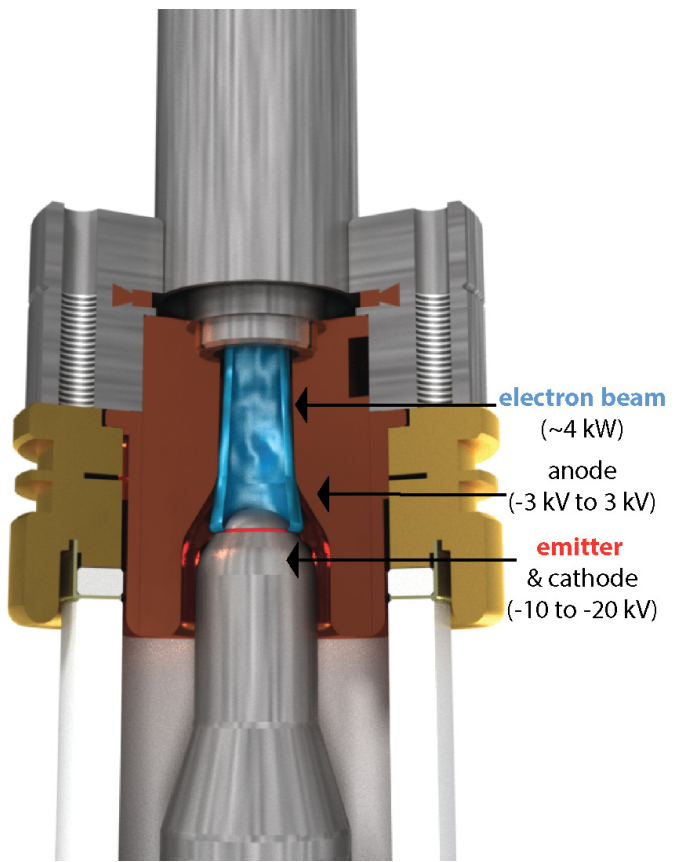

Fig. 7. High power microwave frequency agility scheme. (a) Flow chart showing setup connectivity from the software input to gyrotron connection for frequency modulation. (b) CAD solid model of the electron gun (purchased from Bridge12) indicating the electron beam, anode, cathode housing and the beam emitter.

inversion efficiency of $56 \%$ at $5 \mathrm{kHz}$ and $30 \mathrm{kHz}$. Slow to moderate spinning frequencies seem to have a negligible impact on the inversion efficiency.

\section{Experimental Gyrotron Acceleration Voltage Profiles}

Gyrotrons can provide continuous wave output powers of 10$100 \mathrm{~W}$ and are now commonly employed in high field MAS DNP experiments [67]. Here, we discuss how frequency agile gyrotron oscillators are excellent microwave sources for the implementation of the frequency modulated cross effect, adiabatic EPR inversions, and hyperfine decoupling in MAS DNP. Such fast-tuning gyrotron oscillators for high-field pulsed DNP have advantages over previously proposed gyrotron amplifiers in terms of duty cycle, cost, and the possibility to upgrade DNP NMR spectrometers currently in use.

\subsection{Frequency agile gyrotron oscillators}

In gyrotron oscillators, an electron beam generated by an electron gun deposits microwave energy into an interaction cavity. A heated filament located beneath the surface of an annular barium emitter and a large $(\sim 10-20 \mathrm{kV})$ potential overcome the work function of electrons on the surface of the barium emitter. Electrons ejected from the emitter accelerate under the electric potential between the cathode and the anode
(Fig. 7b) and approach relativistic speeds. In the field of the gyrotron's superconducting magnet, the electrons follow a tight helical path about the magnetic field lines with a cyclotron resonant frequency of:

$$
\Omega_{c}=e B_{0} / m
$$

Where $\Omega_{C}$ is the cyclotron resonant frequency, $e$ is the charge of an electron, and $m$ is the relativistic mass of an electron. The relativistic mass is:

$$
m=\frac{m_{e}}{\sqrt{1-v^{2} / c^{2}}}
$$

where $v$ is the velocity of the electron, $c$ is the speed of light, and $m_{e}$ is the rest mass of the electron.

As the electron beam passes through the cavity of the gyrotron, the high energy electrons deposit energy into the interaction cavity at the cyclotron resonant frequency. The velocity in equation (17) is a function of both the magnetic field and the electric field potential applied across the electron gun to accelerate the electron beam. Therefore, a modulation of the electric potential across the electron gun will lead to a change in the cyclotron resonant frequency and thus provide tuning of the microwave frequency, given the interaction cavity can support continuous frequency tuning [15].

\subsection{Experimental anode potential modulations}

Alberti et al. demonstrated fast frequency control and an instantaneous bandwidth of $>2 \mathrm{GHz}$ from a voltage tunable gyrotron oscillator designed for DNP experiments at $263 \mathrm{GHz}$ $[68,69]$. Idehara et al. recently demonstrated high power levels $(>100 \mathrm{~W})$ during frequency modulations for DNP experiments at $460 \mathrm{GHz}[70,71]$. Following these demonstrations, we have integrated the control of the gyrotron anode potential into the NMR-EPR console (TecMag, TX) to allow for flexible control and synchronization of NMR pulse sequence elements and the rotor period with microwave pulses and EPR control. The NMR console has an arbitrary waveform generator (AWG) with a $10 \mathrm{~ns}$ step size that drives a high voltage amplifier. The high voltage amplifier (TREK, model 5/80) can generate $\pm 5 \mathrm{kV}$ potential with $\pm 80 \mathrm{~mA}$ current and has a slew rate of $1 \mathrm{kV} / \mu \mathrm{s}$. Fig. 7a shows how the anode potential is controlled from the NMR EPR console. This integrated EPR control gives us flexibility to implement the swept voltage profiles shown in Fig. 5d for adiabatic EPR spin inversion of the SA-BDPA lineshape and voltage modulations shown in Fig. $8 \mathrm{a}$ for the frequency modulated cross effect.

We used a frequency dependence on the gyrotron acceleration voltage of $460 \mathrm{MHz} / 1.2 \mathrm{kV}$ to determine the potentials shown in Figs. 5d and 8a [72]. Following Han and colleagues [73], we expect to correct for deviations from this linear response by altering the controlling voltage waveform. Fig. 8a shows the estimated voltage modulations required for the frequency modulated cross effect. Fig. $8 \mathrm{~b}$ overlays predicted microwave frequency bandwidths of different modulation frequencies on the nitroxide lineshape at 7 Tesla calculated with EasySpin [44]. The smaller voltage magnitude of the $500 \mathrm{KHz}$ modulation compared to $10 \mathrm{kHz}$ and $100 \mathrm{kHz}$ reflects the limitation of the microwave frequency bandwidth at higher modulation frequencies due to the slew rate of the TREK HV amplifier. 

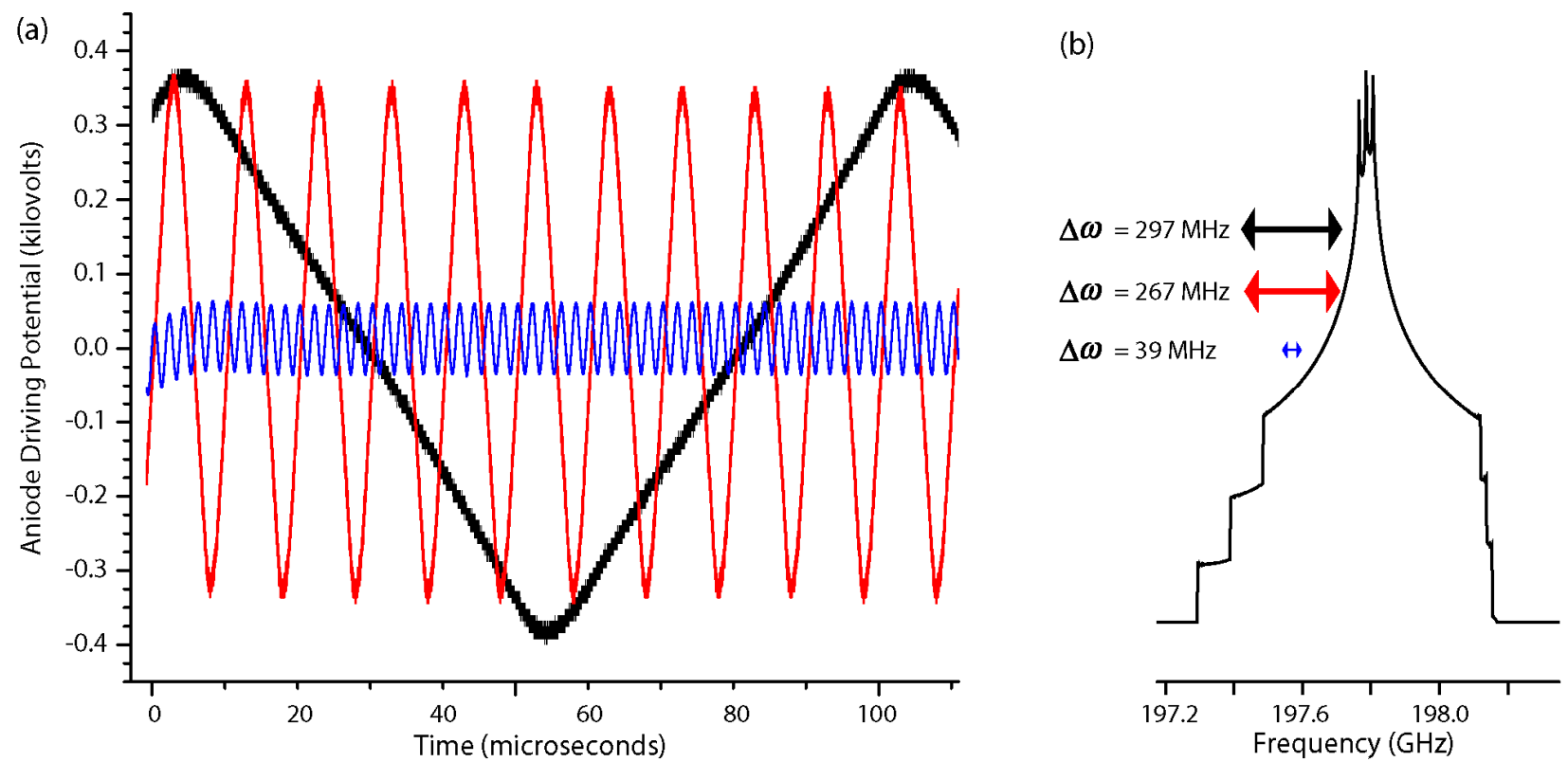

Fig. 8. High power microwave frequency modulation scheme for the cross effect (CE) under MAS. (a) Anode driving potential modulations at selected frequencies of $10 \mathrm{kHz}$ (black), $100 \mathrm{kHz}$ (blue), and $500 \mathrm{kHz}$ (red). (b) Predicted bandwidth of the microwave frequency output arising from modulating the anode potential overlaid on the nitroxide EPR lineshape at 7 Tesla. The EPR spectrum is from EasySpin [44], and was simulated without homogeneous broadening in order to emphasize the ${ }^{14} \mathrm{~N}$ hyperfine couplings.

\section{Conclusions}

We simulated the spatial intensity of microwave fields within a $198 \mathrm{GHz}$ MAS DNP sample. Given these field strengths, we calculated that adiabatic EPR inversions should be possible with currently accessible DNP instrumentation, providing an immediate avenue for broadband hyperfine decoupling in rotating solids. We also show modulations of gyrotron acceleration potentials required to perform both hyperfine decoupling and the frequency modulated cross effect. All together, these results suggest a promising strategy for the implementation of timedomain DNP and hyperfine decoupling in rotating solids.

\section{Acknowledgements}

Research reported in this publication was supported by the National Institute of General Medical Sciences of the National Institutes of Health under Award Number DP2GM119131, the National Cancer Institute of the National Institutes of Health under Award Number P50CA094056, and the National Science Foundation under Award Number STTR-1521314. We thank Mark Conradi, Jagadishwar Sirigiri, Albert Smith, and Marc Caporini for helpful discussions. Computations were performed using the facilities of the Washington University Center for High Performance Computing, which were partially funded by NIH grants 1S10RR022984-01A1 and 1S10OD018091-01. 
18. Gullion, T. and J. Schaefer, Rotational-Echo DoubleResonance NMR. J. Magn. Reson., 1989. 81: p. 196-200.

\section{References}

1. Carver, T.R. and C.P. Slichter, Polarization of Nuclear Spins in Metals. Physical Review, 1953. 92: p. 212-213.

2. Afeworki, M., R.a. Mckay, and J. Schaefer, Dynamic Nuclear-Polarization Enhanced Nuclear-MagneticResonance of Polymer-Blend Interfaces. Materials Science and Engineering a-Structural Materials Properties Microstructure and Processing, 1993. 162(1-2): p. 221228.

3. Afeworki, M. and J. Schaefer, Mechanism of DNPEnhanced Polarization Transfer Across the Interface of Polycarbonate/Polystyrene Heterogeneous Blends. Macromolecules, 1992. 25: p. 4092-4096.

4. Afeworki, M., S. Vega, and J. Schaefer, Direct Electronto-Carbon Polarization Transfer in Homgeneously-Doped Polycarbonates. Macromolecules, 1992. 25: p. 4100-4106.

5. $\quad \mathrm{Ni}$, Q.Z., et al., High frequency dynamic nuclear polarization. Accounts of chemical research, 2013. 46(9): p. 1933-1941.

6. Ravera, E., et al., DNP-enhanced MAS NMR of bovine serum albumin sediments and solutions. The Journal of Physical Chemistry B, 2014. 118(11): p. 2957-2965.

7. Koers, E.J., et al., Dynamic Nuclear Polarization NMR Spectroscopy: Revealing Multiple Conformations in Lipid-Anchored Peptide Vaccines. Angewandte Chemie International Edition, 2013. 52(41): p. 10905-10908.

8. Wylie, B.J., et al., Dynamic nuclear polarization of membrane proteins: covalently bound spin-labels at protein-protein interfaces. Journal of biomolecular NMR, 2015. 61(3-4): p. 361-367.

9. $\quad$ Fernández-de-Alba, C., et al., Matrix-Free DNP-Enhanced NMR Spectroscopy of Liposomes Using a Lipid-Anchored Biradical. Chemistry-A European Journal, 2015. 21(12): p. 4512-4517.

10. Takahashi, H., et al., Solid-state NMR on bacterial cells: selective cell wall signal enhancement and resolution improvement using dynamic nuclear polarization. Journal of the American Chemical Society, 2013. 135(13): p. 5105-5110.

11. Rosay, M., et al., Sensitivity-enhanced NMR of biological solids: Dynamic nuclear polarization of Y21M $\mathrm{fd}$ bacteriophage and purple membrane. Journal of the American Chemical Society, 2001. 123(5): p. 1010-1011.

12. Hu, K.-N., et al., Dynamic nuclear polarization with biradicals. Journal of the American Chemical Society, 2004. 126(35): p. 10844-10845.

13. Kubicki, D.J., et al., Amplifying Dynamic Nuclear Polarization of Frozen Solutions by Incorporating Dielectric Particles. Journal of the American Chemical Society, 2014. 136(44): p. 15711-15718.

14. Becerra, L.R., et al., Dynamic Nuclear-Polarization with a Cyclotron-Resonance Maser at 5-T. Physical Review Letters, 1993. 71(21): p. 3561-3564.

15. Torrezan, A.C., et al., Continuous-Wave Operation of a Frequency-Tunable 460-GHz Second-Harmonic Gyrotron for Enhanced Nuclear Magnetic Resonance. IEEE Transactions on Plasma Science, 2010. 38(6): p. 11501159.

16. Barnes, A.B., et al., Dynamic nuclear polarization at 700 $\mathrm{MHz} / 460 \mathrm{GHz}$. Journal of Magnetic Resonance, 2012. 224: p. 1-7.

17. Kaplan, M., et al., Probing a cell-embedded Megadalton protein complex by DNP-supported solid-state NMR. Nature methods, 2015.
19. Hing, A.W., S. Vega, and J. Schaefer, Transferred-echo double-resonance NMR. Journal of Magnetic Resonance (1969), 1992. 96(1): p. 205-209.

20. Un, S., et al., Pulsed dynamic nuclear polarization at $5 \mathrm{~T}$. Chemical physics letters, 1992. 189(1): p. 54-59.

21. Can, T., Q. Ni, and R. Griffin, Mechanisms of dynamic nuclear polarization in insulating solids. Journal of Magnetic Resonance, 2015. 253: p. 23-35.

22. Hovav, Y., et al., Dynamic nuclear polarization using frequency modulation at $3.34 \mathrm{~T}$. Journal of Magnetic Resonance, 2014. 238: p. 94-105.

23. Bornet, A., et al., Microwave frequency modulation to enhance dissolution dynamic nuclear polarization. Chemical Physics Letters, 2014. 602: p. 63-67.

24. Henstra, A., et al., High Dynamic Nuclear Polarization at Room Temperature. J. Magn. Reson., 1988. 77: p. 389.

25. Henstra, A., et al., Nuclear Spin Orientation via Electron Spin Locking (NOVEL). J. Magn. Reson., 1988. 77: p. 389393.

26. Henstra, A., P. Dirksen, and W.T. Wenckebach, Enhanced Dynamic Nuclear Polarization by the Integrated Solid Effect. Phys. Letters, 1988. A134: p. 134.

27. Henstra, A. and W.T. Wenckebach, Dynamic nuclear polarisation via the integrated solid effect I: theory. Molecular Physics, 2014. 112(13): p. 1761-1772.

28. Eichhorn, T., et al., Dynamic nuclear polarisation via the integrated solid effect II: experiments on naphthalene-h 8 doped with pentacene-d 14. Molecular Physics, 2014. 112(13): p. 1773-1782.

29. Can, T., et al., Time domain DNP with the NOVEL sequence. The Journal of chemical physics, 2015. 143(5): p. 054201.

30. Schaefer, J. and E.O. Stejskal, ${ }^{13} \mathrm{C}$ Nuclear Magnetic Resonance of Polymers Spinning at the Magic Angle. J. Am. Chem. Soc., 1976. 98: p. 1031-1032.

31. Thurber, K.R. and R. Tycko, Perturbation of nuclear spin polarizations in solid state NMR of nitroxide-doped samples by magic-angle spinning without microwaves. The Journal of chemical physics, 2014. 140(18): p. 184201.

32. Lange, S., et al., The effect of biradical concentration on the performance of DNP-MAS-NMR. Journal of Magnetic Resonance, 2012. 216: p. 209-212.

33. Corzilius, B., et al., Paramagnet induced signal quenching in $M A S-D N P$ experiments in frozen homogeneous solutions. Journal of Magnetic Resonance, 2014. 240: p. 113-123.

34. Jeschke, G. and A. Schweiger, Hyperfine decoupling in electron spin resonance. The Journal of chemical physics, 1997. 106(24): p. 9979-9991.

35. Du, J., et al., Preserving electron spin coherence in solids by optimal dynamical decoupling. Nature, 2009. 461(7268): p. 1265-1268.

36. Dolde, F., et al., High-fidelity spin entanglement using optimal control. Nature communications, 2014. 5.

37. Ramanathan, C., Dynamic nuclear polarization and spin diffusion in nonconducting solids. Applied Magnetic Resonance, 2008. 34(3-4): p. 409-421.

38. Wolfe, J.P., Direct Observation of a Nuclear Spin Diffusion Barrier. Physical Review Letters, 1973. 31(15): p. 907-910.

39. Nanni, E.A., et al., Microwave field distribution in a magic angle spinning dynamic nuclear polarization NMR probe. Journal of Magnetic Resonance, 2011. 210(1): p. 16-23.

40. Macor, A., et al., THz-waves channeling in a monolithic saddle-coil for Dynamic Nuclear Polarization enhanced NMR. Journal of Magnetic Resonance, 2011. 212(2): p. 440-449. 
41. Thurber, K.R. and R. Tycko, Theory for cross effect dynamic nuclear polarization under magic-angle spinning in solid state nuclear magnetic resonance: The importance of level crossings. The Journal of chemical physics, 2012. 137(8): p. 084508.

42. Mance, D., et al., The magnetic field dependence of crosseffect dynamic nuclear polarization under magic angle spinning. The Journal of chemical physics, 2015. 142(23): p. 234201 .

43. Corzilius, B., A.A. Smith, and R.G. Griffin, Solid effect in magic angle spinning dynamic nuclear polarization. The Journal of chemical physics, 2012. 137(5): p. 054201.

44. Stoll, S. and A. Schweiger, EasySpin, a comprehensive software package for spectral simulation and analysis in $E P R$. Journal of Magnetic Resonance, 2006. 178(1): p. 4255.

45. Siaw, T.A., et al., Effect of electron spin dynamics on solid-state dynamic nuclear polarization performance. Physical Chemistry Chemical Physics, 2014. 16(35): p. 18694-18706.

46. Sakamoto, K., et al., Achievement of robust high-efficiency $1 \mathrm{MW}$ oscillation in the hard-self-excitation region by a $170 \mathrm{GHz}$ continuous-wave gyrotron. Nature physics, 2007. 3(6): p. 411-414.

47. Tax, D.S., et al., Experimental Results for a Pulsed 110 GHz/124.5 GHz Megawatt Gyrotron. 2013.

48. Smith, A.A., et al., A $140 \mathrm{GHz}$ pulsed EPR/212MHz NMR spectrometer for DNP studies. Journal of Magnetic Resonance, 2012. 223: p. 170-179.

49. Neugebauer, P., et al., Liquid state DNP of water at $9.2 \mathrm{~T}$ : an experimental access to saturation. Physical Chemistry Chemical Physics, 2013. 15(16): p. 6049-6056.

50. Reginsson, G.W., et al., Trityl Radicals: Spin Labels for Nanometer-Distance Measurements. Chemistry-A European Journal, 2012. 18(43): p. 13580-13584.

51. Mitchell, D.G., et al., Electron spin relaxation and heterogeneity of the 1: $1 \alpha, \gamma$-bisdiphenylene- $\beta$-phenylallyl (BDPA)/benzene complex. The Journal of Physical Chemistry B, 2011. 115(24): p. 7986-7990.

52. Michaelis, V.K., et al., High-Field 13C Dynamic Nuclear Polarization with a Radical Mixture. Journal of the American Chemical Society, 2013. 135(8): p. 2935-2938.

53. Smith, A.A., et al., Solid effect dynamic nuclear polarization and polarization pathways. The Journal of chemical physics, 2012. 136(1): p. 015101.

54. Smith, A.A., et al., Observation of strongly forbidden solid effect dynamic nuclear polarization transitions via electron-electron double resonance detected NMR. The Journal of chemical physics, 2013. 139(21): p. 214201.

55. Can, T., et al., Overhauser effects in insulating solids. The Journal of chemical physics, 2014. 141(6): p. 064202.

56. Haze, O., et al., Water-Soluble Narrow-Line Radicals for Dynamic Nuclear Polarization. Journal of the American Chemical Society, 2012. 134(35): p. 14287-14290.

57. Kupce, E. and R. Freeman, Adiabatic pulses for wideband inversion and broadband decoupling. Journal of Magnetic Resonance, Series A, 1995. 115(2): p. 273-276.

58. Garwood, M. and L. DelaBarre, The return of the frequency sweep: designing adiabatic pulses for contemporary NMR. Journal of Magnetic Resonance, 2001. 153(2): p. 155-177.

59. Kupce, E. and R. Freeman, Optimized adiabatic pulses for wideband spin inversion. Journal of Magnetic Resonance, Series A, 1996. 118(2): p. 299-303.

60. Fu, R. and G. Bodenhausen, Broadband decoupling in NMR with frequency-modulated 'chirp'pulses. Chemical physics letters, 1995. 245(4): p. 415-420.

61. Starcuk, Z. and K. Bartusek, Heteronuclear broadband spin-flip decoupling with adiabatic pulses. Journal of Magnetic Resonance, Series A, 1994. 107(1): p. 24-31.
62. Hwang, T.-L., P.C. van Zijl, and M. Garwood, Fast broadband inversion by adiabatic pulses. Journal of Magnetic Resonance, 1998. 133(1): p. 200-203.

63. Schmidt, M.W., et al., General atomic and molecular electronic structure system. Journal of computational chemistry, 1993. 14(11): p. 1347-1363.

64. Veshtort, M. and R.G. Griffin, SPINEVOLUTION: a powerful tool for simulations of solid and liquid state NMR experiments. Journal of Magnetic Resonance, 2006. 178: p. 248-282

65. Smith, A.A., Personal communication. 2015.

66. Hogben, H., et al., Spinach-a software library for simulation of spin dynamics in large spin systems. Journal of Magnetic Resonance, 2011. 208(2): p. 179-194.

67. Rosay, M., et al., Solid-state dynamic nuclear polarization at $263 \mathrm{GHz}$ : spectrometer design and experimental results. Phys. Chem. Chem. Phys., 2010. 12(22): p. 5850-5860.

68. Alberti, S., et al., Experimental study from linear to chaotic regimes on a terahertz-frequency gyrotron oscillator. Physics of Plasmas (1994-present), 2012. 19(12): p. 123102.

69. Alberti, S., et al., Nanosecond pulses in a THz gyrotron oscillator operating in a mode-locked self-consistent $Q$ switch regime. Physical review letters, 2013. 111(20): p. 205101.

70. Ikeda, R., et al., Broadband continuously frequency tunable gyrotron for $600 \mathrm{MHz}$ DNP-NMR spectroscopy. Plasma and Fusion Research: Rapid Communications, 2014. 9: p. 1206058.

71. Idehara, T., et al., High-Speed Frequency Modulation of a 460-GHz Gyrotron for Enhancement of 700-MHz DNPNMR Spectroscopy. Journal of Infrared, Millimeter, and Terahertz Waves, 2015: p. 1-11.

72. Barnes, A.B., et al., A $250 \mathrm{GHz}$ Gyrotron With a $3 \mathrm{GHz}$ Tuning Bandwidth for Dynamic Nuclear Polarization. Journal of Magnetic Resonance, 2012. 221: p. 147-153.

73. Kaufmann, T., et al., DAC-board based X-band EPR spectrometer with arbitrary waveform control. Journal of Magnetic Resonance, 2013. 235: p. 95-108. 